improving information regarding to the work-related diseases and, more specifically, on the trend and variation of benefits paid for them.

Method Through the NTEP it is possible to establish a nexus for each area of the economic activity, focusing on data of incapacitating diseases recognised by social welfare and involving more than 15 days' absence from work, using the ICD-10.

Results Benefits for work-related diseases increased 128.2\% during 2005-2008. However, the greatest changes occurred after 2007. From May 2006 to March 2007, when only the employer's CAT was used, 125246 Accident and Disease Assistance authorizations were issued. But, with the addition of the NTEP to the CAT this number rose to 293912 , an increase of $134.7 \%$ over the period from April 2007 to February 2008. The detachable figures are for "musculoskeletal system and connective tissue" (107 764 cases), "mental and behavioural disorders" (8930 cases), "and "diseases of the nervous system" (8396 cases).

Conclusions The accident benefits for work-related diseases are growing more than other welfare benefits. This reality requires more studies and technical insights as well as priorities in terms of specific strategies for OSH policy.

\section{ASSOCIATIONS BETWEEN JOB STRESS, SOCIAL SUPPORT AND INSOMNIA AMONG NURSES}

${ }^{1}$ Caroline Kröning Luna, ${ }^{1}$ Lúcia Rotenberg, ${ }^{1}$ Aline Silva-costa, ${ }^{2}$ Susanna Toivanen, ${ }^{3}$ Tânia Araújo, 'Luciana Fernandes Portela, ${ }^{1}$ Rosane Härter Griep. ${ }^{1}$ Education Laboratory for Environment and Health (LEAS), Instituto Oswaldo Cruz - Fiocruz, Rio de Janeiro, Brazil; ${ }^{2}$ Centre for Health Equity Studies, StockholmUniversity/Karolinska Institute, Stockholm, Sweden; ${ }^{3}$ Health Department, State University of Feira de Santana, Feira de Santana, Brazil

\subsection{6/oemed-2014-102362.249}

Objectives To investigate the association between job strain, social support at work and insomnia among registered nurses.

Method A cross-sectional study was conducted among 3.229 nurses $(87 \%$ women) in 18 major public hospitals in Rio de Janeiro/Brazil. Data collection was based on a comprehensive self-filled questionnaire that included questions on insomnia and the Job Content Questionnaire (JCQ). Job strain (high psychological demands and low control) and social support were evaluated by the Portuguese version of the 2.0-JCQ, defined by the quadrant approach. In addition, emotional demands were also assessed, so that high strain was evaluated considering separately the psychological and the emotional demands. Insomnia was defined as having at least one of the insomnia symptoms: difficulty initiating sleep, maintaining sleep or early morning awakening. Multivariate logistic regression and adjusted odds ratios and 95\% confidence intervals were calculated.

Results The overall prevalence of insomnia was 34.3\%. Individuals with high job strain and low social support experienced insomnia more frequently $(\mathrm{p}<0.05)$. After adjusting for sociodemographic, work and health-related variables, high strain doubled the chances of presenting insomnia considering both the psychological $(\mathrm{OR}=2.20, \mathrm{CI} 1.74-2.78)$ and the emotional demands $(\mathrm{OR}=1.99$, CI 1.57-2.53). High strain in combination with low support at work increased the chances of insomnia even more.

Conclusions High strain is suggested as a possible risk factor for insomnia considering both psychological and emotional demands. The lack of social support from co-workers and supervisors seem to potentiate the odds for insomnia.

\section{THE RELATIONSHIP BETWEEN LOW LEVEL BENZENE EXPOSURE AND BLOOD CELL COUNTS IN KOREAN WORKERS}

${ }^{1}$ Dong-Hee Koh, ${ }^{2}$ Hee-Kyung Cheon, ${ }^{2}$ Hyang-Woo Ryu, ${ }^{2}$ Sang-Gil Lee. ${ }^{1}$ Carcinogenic Hazard Brach, National Cancer Control Institute, National Cancer Center, Goyang-Si, Gyeonggi-Do, Republic of Korea; ${ }^{2}$ Occupational Safety and Health Research Institute, Korea Occupational Safety and Health Agency, Incheon, Republic of Korea

\subsection{6/oemed-2014-102362.250}

Objectives Benzene is a well-known haematological toxin causing aplastic anaemia and leukaemia. Recent studies showed that low level benzene less than 1ppm disturbs the hematopoietic system. However, other studies did not show consistent results. The aim of the present study was to examine the relationship between low level benzene exposure and blood cell counts in Korean workers.

Method Blood cell counts of benzene exposed workers were retrieved from a nationwide Worker's Health Examination Database from 2003 to 2008. If a worker did not take a blood test for benzene during 2003-2004, the worker was regarded as a first exposed. Personal air benzene monitoring records were retrieved from nationwide Work Environment Monitoring Database from 2004-2008. Mean benzene levels were calculated and assigned for the various combinations of factory/industry/process codes. Mixed-effects models were employed to examine associations between benzene level, and the numbers of WBC, RBC, platelet, segmented neutrophil, lymphocyte and monocyte.

Results 11281 workers took blood tests during 2005-2008 for the first time. 8679 personal benzene measurements during 2004-2008 across industries were collected. RBC counts showed a significant negative association with low level benzene exposure with a dose-response relationship. WBCs also showed negative association, but did not show a dose-response relationship. Among WBCs, lymphocyte showed a stronger association with low level benzene than other cell types.

Conclusions Our findings support the hematotoxicity of low level benzene exposure. Further study with direct benzene measuring for first exposed workers is needed to confirm the low level benzene toxicity in Korean workers.

\section{DEVELOPMENT OF AN INSTRUMENT ASSESSING SYMPTOM EXAGGERATION IN PATIENTS RECEIVING DISABILITY BENEFITS SECONDARY TO MENTAL HEALTH DISORDERS}

1,2 2 hanil Ebrahim, ${ }^{3}$ Sheena Bance, ${ }^{4}$ Luis Montoya, ${ }^{1}$ Sohail Mulla, ${ }^{1}$ Mostafa Kamal el Din, ${ }^{3}$ Cindy Malachowski, 'Gordon Guyatt, ${ }^{1}$ Busse. ${ }^{1}$ McMaster University, Hamilton, Canada; ${ }^{2}$ Stanford University, Stanford, USA; ${ }^{3}$ University of Toronto, Toronto, Canada; ${ }^{4}$ University Health Network, Toronto, Canada

\subsection{6/oemed-2014-102362.251}

Objectives Symptom exaggeration is a significant issue in patients receiving disability benefits secondary to mental health disorders. Measures designed to detect exaggeration of symptoms are valuable for informing more accurate diagnoses, which can impact claim decision-making, both for disability claim approval and patient management. Our objectives were: 1) to complete a systematic review to identify measures that assess symptom exaggeration in mental health disorders, and 2) using the results from the review, develop an instrument assessing symptom exaggeration in individuals receiving disability benefits secondary to mental health disorders. 
Method We completed searches of MEDLINE and PSYCInfo from inception of each database to June 2013, to identify primary studies evaluating symptom exaggeration among patients with mental health disorders. Teams of reviewers completed title and abstract screening of citations, full text review of potentially eligible articles, data extraction, and quality assessment of eligible studies. Findings from our review and input from content experts informed the development of a summary instrument.

Results We identified 8435 unique citations; 102 studies were eligible. Studies reported on 55 different instruments to assess symptom exaggeration; $42 \%$ of studies used the MMPI-2. Quality of studies ranged from low to moderate. Items that comprise our summary instrument will be presented at EPICOH.

Conclusions Multiple instruments are available to assess symptom exaggeration among patients presenting with mental illness; however, no instrument has shown ideal psychometric properties. We are hopeful that our instrument will facilitate the testing and development of a novel tool with superior sensitivity and specificity for detecting symptom exaggeration.

\section{DEVELOPMENT OF AN INSTRUMENT ASSESSING RECOVERY EXPECTATIONS IN PATIENTS RECEIVING DISABILITY BENEFITS SECONDARY TO MENTAL HEALTH DISORDERS}

1,2Shanil Ebrahim, ${ }^{3}$ Cindy Malachowski, ${ }^{1}$ Mostafa Kamal el Din, ${ }^{1}$ Sohail Mulla, ${ }^{3}$ Sheena Bance, ${ }^{4}$ Luis Montoya, ${ }^{1}$ Gordon Guyatt, ${ }^{1} J a s o n$ Busse. ${ }^{1}$ McMaster University, Hamilton, Canada; ${ }^{2}$ Stanford University, Stanford, USA; ${ }^{3}$ University of Toronto, Toronto, Canada; ${ }^{4}$ University Health Network, Toronto, Canada

\subsection{6/oemed-2014-102362.252}

Objectives Of workers approved for long-term disability benefits, $31.6 \%$ suffer from a primary mental illness. Negative patient recovery expectations are associated with worse outcome in many conditions. Our objectives were: 1) to complete a systematic review to identify measures that assess patient recovery expectations, and 2) using the results from our review, develop an instrument designed to assess recovery expectations in individuals receiving disability benefits secondary to a mental health disorder.

Method We completed searches of MEDLINE and PSYCInfo from inception of each database to June 2013, to identify primary studies evaluating patient's recovery expectations across all clinical populations. Teams of reviewers completed title and abstract screening of citations, full text review of potentially eligible articles, data extraction, and quality assessment of eligible studies. Findings from the review and input from content experts informed the development of a comprehensive list of items that captured patient's recovery expectations.

Results We identified 12599 unique citations; 46 studies were eligible. Studies reported on 20 different instruments assessing patient's recovery expectations. A minority (20\%) of studies included measurement properties of reported instruments; 11\% evaluated mental health conditions. No instruments were validated among patients receiving disability benefits or explored associations with return to work. Generalizability to disabled patients receiving wage replacement benefits is therefore unclear. Items that comprise our summary instrument will be presented at $\mathrm{EPICOH}$.

Conclusions Our instrument holds promise for identifying claimants holding negative recovery expectations, which may be associated with prolonged recovery. These unhelpful beliefs may be a useful target for early interventional therapies.

\section{SHIFT WORK AND HYPERTENSION: PREVALENCE AND ANALYSIS OF DISEASE PATHWAYS IN GERMAN CAR MANUFACTURING WORKERS}

${ }^{1}$ Johan Ohlander, ${ }^{2}$ Mekail-Cem Keskin, ${ }^{2}$ Joachim Stork, ${ }^{1}$ Katja Radon. ${ }^{1}$ University Hospital Munich, Munich, Bavaria, Germany; ${ }^{2}$ AUDI AG, Ingolstadt, Bavaria, Germany

\subsection{6/oemed-2014-102362.253}

Objectives Triggered by disturbed circadian rhythm, increased blood pressure comprises an intermediate step on suggested pathways between shift work and cardiovascular disease. Despite accumulated evidence indicating an excess risk for cardiovascular disease among shift workers, studies on shift work and hypertension are inconclusive. Thus, we investigated the association between shift work and hypertension in German car manufacturing workers.

Method Baseline blood pressure and potential confounders for 25343 workers aged 16 to 64 years were obtained from standardised medical check-ups. Workers were analysed according to four exposure categories: no shift work, shift work without night shifts, shift work with night shifts and constant night shifts. Cross-sectional associations between shift work and hypertension were analysed using logistic regression adjusted for confounders and sets of behavioural, psychosocial and physiological factors.

Results Hypertension prevalence was highest among shift workers not working night shifts (11.5\%), and lowest among workers not working shifts $(7.8 \%)$. Unadjusted, a significant positive association with hypertension was found for shift work without night shifts (OR 1.53 95\% CI 1.40-1.68) and constant night shifts (OR 1.46, 95\% CI 1.29-1.65). Adjusted for confounders, only shift work without night shifts showed an increased hypertension risk (OR 1.15, 95\% CI 1.02-1.30). However, stratification for occupational status suggested this excess risk to be attributable only to white-collar shift workers not working night shifts (OR 1.52 95\% CI 1.22-1.88). After adjustments for behavioural factors no shift work category showed increased hypertension risks.

Conclusions Shift workers' increased hypertension risk might be explained by behavioural risk factors, possibly triggered by disturbed circadian rhythm.

\section{PRELIMINARY RESULTS OF SHIFT WORK AND CARDIOVASCULAR RISK FACTORS: ANALYSING BASELINE DATA OF A PROSPECTIVE NIGHT SHIFT WORKER COHORT IN SHENZHEN, CHINA}

\footnotetext{
1,2 Shelly Lap Ah Tse, ${ }^{1,2}$ Feng Wang, ${ }^{3}$ Liuzhuo Zhang, ${ }^{3}$ Yanfang Zhang, ${ }^{1}$ Bo Zhang, ${ }^{1,4}{ }^{4}$ Yonghua He, 'Shaohua Xie, ${ }^{1}$ Mengjie Li, ${ }^{3}$ Zhimin Li, 'Ignatius Tak-sun Yu. ${ }^{1} \mathrm{JC}$ School of Public Health and Primary Care, The Chinese University of Hong Kong, Sha Tin, Hong Kong; ${ }^{2}$ Shenzhen Municipal Key Laboratory for Health Risk Analysis, Shenzhen Research Institute of the Chinese University of Hong Kong, Shenzhen, China; ${ }^{3}$ Shenzhen Prevention and Treatment Center for Occupational Diseases, Shenzhen, China; ${ }^{4}$ CUHK Centre for Public Health and Primary Care (Shenzhen), Shenzhen Research Institute of the Chinese University of Hong Kong, Shenzhen, China
}

\subsection{6/oemed-2014-102362.254}

Objectives The incidences of cardiovascular diseases (CVD), diabetes mellitus and metabolic syndrome increase rapidly in China, while the prevalence of night shift work is also increasing. This study aims to investigate the relationship between night shift work and CVD risk factors by analysing the baseline data of a prospective cohort study. 